\title{
Prospects for Treatment of Latent HIV
}

\author{
KM Barton ${ }^{1}$, BD Burch ${ }^{2}$, N Soriano-Sarabia ${ }^{2}$, and DM Margolis ${ }^{1,2,3}$ \\ ${ }^{1}$ Department of Microbiology and Immunology, University of North Carolina at Chapel Hill, Chapel \\ Hill, North Carolina, USA \\ ${ }^{2}$ Department of Medicine, University of North Carolina at Chapel Hill, Chapel Hill, North Carolina, \\ USA \\ ${ }^{3}$ Department of Epidemiology, University of North Carolina at Chapel Hill, Chapel Hill, North \\ Carolina, USA
}

\begin{abstract}
Recent advances in antiretroviral therapy (ART) have drastically improved the quality of life for people with HIV infection. However, owing to the persistence of latent HIV in the presence of therapy, patients must remain on therapy indefinitely. Currently, the solution to the HIV pandemic rests on the prevention of new infections and many decades of ART for the steadily expanding number of people infected worldwide. ART is costly, requires ongoing medical care, and can have side effects, thereby preventing its universal availability. Therefore, to escape the ironic burdens of therapy, efforts have begun to develop treatments for latent HIV infection. Current approaches propose either complete eradication of infection or induction of a state of stringent control over viral replication without ART. This review will discuss these strategies in detail and their potential for clinical development.
\end{abstract}

More than 30 million people are currently infected with HIV, and more than two million became newly infected in 2010. ${ }^{1}$ Significant progress has been made in the treatment of HIV infection, and current therapies allow infected individuals to attain near-normal life spans. However, antiretroviral therapy (ART) is costly and not readily available throughout the world, making HIV a significant economic drain and a major driver of morbidity and mortality around the globe. Although current HIV therapies effectively diminish HIV viral loads to undetectable levels, a small population of quiescent but replication-competent viruses persists for years in viral reservoirs such as resting $\mathrm{CD} 4^{+} \mathrm{T}$ cells and perhaps in other cell types. On discontinuation of ART, these viruses can reactivate and lead to waves of de novo infection events. Therefore, to relieve the unsustainable burden of lifelong ART for millions of people, the need for curative therapies has recently attained the status of "research priority."

Quiescent or latent viruses escape the effects of ART by integrating into the host genome and becoming transcriptionally silent. The two primary defenses that patients have against viral replication are the immune system and ART. ART regimens target several stages of the viral life cycle, including viral entry, reverse transcription, integration, and virion assembly. However, virus that integrates into the genome of the host is indistinguishable from the host's DNA and is therefore very difficult to target. Cells containing latent viral integrants

(C) 2012 American Society for Clinical Pharmacology and Therapeutics

Correspondence: DM Margolis (dmargo@med.unc.edu).

CONFLICT OF INTEREST

The other authors declared no conflict of interest. 
are not recognized by the immune system, and the virus in these cells does not advance through the stages of the viral life cycle that are targeted by ART. Therefore, proviral quiescence facilitates evasion of both the immune system and ART.

\section{OPTIMAL LATENT HIV THERAPY}

Until recently, few researchers were in serious pursuit of strategies to cure HIV infection, but this effort has recently gained widespread acceptance and attention. Because of this, many novel strategies have been proposed to complement traditional drug therapies. However, regardless of the approach, not all therapies are created equal. Indeed, for a therapy to be effective and feasible for worldwide use, it will have to meet several requirements. First and most important, the therapy must be safe and have manageable side effects. Second, it must not extensively activate the immune system, given that activated T cells are more susceptible to HIV infection and are more difficult to protect with ART. Third, the treatment must have a finite duration that will allow the patient to live a healthy life without the need for ongoing treatment. Fourth, if the goal is eradication, the treatment must be able to access all reservoirs of persistent infection throughout the body. Finally, to ensure global utility and availability, the therapy must be economically and logistically accessible to the developing world, given that this is where the burden of lifelong therapy is least sustainable. Initially, drugs that have already been pharmacologically characterized and approved for use in humans for treatment of other diseases will have an advantage over newer approaches, given that the time from initial testing to implementation will be drastically reduced. That said, many novel approaches to treating HIV infection, some of which are detailed in this review, are receiving a great deal of attention from the research community and may prove invaluable in our pursuit of a cure.

\section{INTRODUCTION TO ERADICATION}

Eradication is what most often comes to mind when people think about a "cure" for HIV. The goal of this approach is clearance of all replication-competent virus from the patient. Purging HIV provirus from latent reservoirs is crucial to any HIV eradication strategy, and interest in this area has spurred major efforts to identify therapeutics that can do so. Most eradication studies have focused on identifying small-molecule drugs that elicit proviral expression with the notion that ART will prevent new infections while the immune system, possibly with the help of other therapeutics, will clear infected cells. Indeed, several smallmolecule drugs that have been developed for the treatment of HIV latency are currently in clinical trials. Beyond these drugs, other conceptual options for eradication include gene therapy approaches such as HIV-specific recombinases that destroy proviral DNA, or HIVdependent suicide genes that selectively kill HIV-infected cells.

\section{INDUCTION AND CLEARANCE STRATEGIES: "KICK AND KILL"}

\section{Background}

Once integrated into the host genome, replication-competent viral DNA can become quiescent, with its expression durably suppressed through the development of repressive chromatin structures on the proviral promoter or the long terminal repeat (LTR). ${ }^{2}$ The goal of induction and clearance strategies, which are also known as "kick and kill" strategies, is to induce transcription of these quiescent, replication-competent HIV proviruses (the "kick"), making them susceptible to immune clearance and the effects of ART (the "kill"). Several compounds are currently being studied that activate transcription of HIV through a variety of mechanisms. 


\section{Histone deacetylase inhibitors}

Histone deacetylase inhibitors (HDACis) were identified as candidate antilatency drugs following the discovery that their target HDACs were specifically recruited to the HIV promoter and maintained latency. ${ }^{3,4}$ Corroborating this, HDACis were identified in several screens for compounds that induce transcription from quiescent HIV proviruses. ${ }^{5}$ Although HDACs might be better termed lysine deacetylases, given that they can remove acetyl groups from both histone and nonhistone proteins, their removal of acetyl groups from the lysine residues within histone tails is associated with reduced transcription from cellular and viral promoters. Inhibition of HDACs allows histone lysine acetylation to persist, which then results in the recruitment of transcriptional activators and facilitates transcription from the HIV promoter (Figure 1). Vorinostat (VOR or suberoylanilide hydroxamic acid) and valproic acid (VPA) are HDACis that activate HIV transcription in cell-line models of latent HIV and in ex vivo cultured patient cells. Both drugs are currently approved for clinical use, VOR for oncology and VPA for a variety of indications, and many of their pharmacological characteristics are known. HIV-centered clinical trials have been conducted for VPA, and two such clinical trials are ongoing for VOR. ${ }^{6,7}$ Previous studies testing VPA using varying designs and end points have had mixed results, but uniform and exposure-dependent depletion of the latent viral reservoir has not been observed. ${ }^{6,8}$ Recent work suggests that alternative HDACis, such as VOR, are able to induce expression from the HIV promoter at lower concentrations than VPA. ${ }^{9}$ Notably, the initial results from the VOR clinical trials are promising. In a trial being conducted by our group, HIV-positive patients were given a 400$\mathrm{mg}$ dose of VOR, and resting $\mathrm{CD} 4^{+} \mathrm{T}$ cells were isolated to measure viral RNA production and global histone $\mathrm{H} 3$ acetylation to determine whether VOR is able to upregulate viral transcription. To date, all patients in the study have demonstrated increased viral RNA production and histone $\mathrm{H} 3$ acetylation, which indicates that HIV latency was disrupted following a single 400-mg dose of VOR (clinical trial NCT01319383) (Table 1). ${ }^{7}$ A second study at the Monash University in Melbourne is under way to assess a multidose regimen of VOR administered at a dose of $400 \mathrm{mg}$ daily for 14 days (Table 1 ). ${ }^{10}$ The results from this trial are currently pending. If the results from these studies verify the ability of VOR to activate quiescent HIV in patients, the next step will be to determine whether this activation has an effect on the viral reservoir.

In addition to VOR and VPA, several additional HDACis that activate transcription of quiescent proviruses are currently in phase II clinical trials for treatment of cancers or juvenile arthritis; these include belinostat, panobinostat, givinostat, and entinostat. ${ }^{9,11}$ Furthermore, a clinical trial for juvenile arthritis in which children ranging from 6 to 17 years of age were administered daily givinostat revealed only minor adverse drug effects, suggesting the potential utility of the drug for treating youth and adolescents. ${ }^{12}$ In addition to activation of HIV, givinostat and VOR have also been demonstrated to transiently reduce expression of a subset of cytokines at a clinically relevant dose in ex vivo studies. ${ }^{13,14}$ Because cytokine production may activate the immune system, making cells susceptible to HIV infection, suppression of cytokine production may prevent infection of additional cells upon reactivation. Depending on the safety of these drugs and their pharmacological suitability, these drugs may also be tested in future clinical trials addressing HIV latency.

Conceptually, it is important to note that the most desirable qualities of the ideal HDACi for clinical application in HIV remain to be defined. Obviously, such a drug must be clinically well tolerated. Current evidence suggests that inhibitors selective for the class I HDACs 1, 2, and 3 are the most relevant,, ${ }^{4}, 15$ and recent studies suggest that HDAC 3 may be the most important target (K.M. Barton and D.M. Margolis, unpublished data). However, highly selective inhibitors for HDAC3 are not available. Although it is widely assumed that a more potent HDAC inhibitor will have a better clinical effect, this is totally unproven. Of central 
importance, the optimal duration and schedule for dosing that is required to achieve sufficient HIV induction without impairing the immune response or inducing toxicity is unknown.

\section{Disulfiram}

Disulfiram is a zinc-chelating agent that is approved for use in humans to treat alcoholism. Its toxicities are manageable. Dilsulfiram and its metabolite diethyldithiocarbamate were recently demonstrated to activate HIV transcription from a primary cell model of latent HIV. ${ }^{16}$ A study by Doyon and colleagues suggests that disulfiram causes depletion of phosphatase and tensin homolog, which is an inhibitor of the Akt pathway. Once inhibition of Akt is removed, it phosphorylates hexamethylene bisacetamide (HMBA) inducible 1, which releases positive transcription elongation factor $b$. Free positive transcription elongation factor $\mathrm{b}$ is then recruited to the HIV promoter, where it can activate transcription (Figure 1). In further support of this model, disulfiram-mediated activation of quiescent HIV proviruses was blocked by an inhibitor of the Akt pathway, which indicates that disulfiram may modulate HIV expression through its effects on phosphatase and tensin homolog and the Akt pathway. ${ }^{17}$ A clinical trial (NCT01286259) with disulfiram has been undertaken to determine its effect on persistent HIV infection in patients. The pilot study enrolled 14 participants who took $500 \mathrm{mg}$ of disulfiram daily for 14 days (Table 1). The initial results from the pilot study revealed a modest but statistically significant increase in plasma HIV RNA in the group as a whole, largely driven by transient and temporally dispersed increases in viral load in only a few patients and a minimal absolute decrease in the frequency of infected resting $\mathrm{CD}^{+} \mathrm{T}$ cells that was within the range of variation seen in viral outgrowth assays. ${ }^{18}$ At this time, it is unclear whether disulfiram can contribute to clinically significant reductions in the viral reservoir.

\section{PD-1 inhibition}

Programmed cell death protein 1 (PD-1), a receptor best known for its role in immune exhaustion, is currently the focus of several studies aimed at treating latent HIV. PD-1 antibodies are being investigated for their ability to activate HIV transcription and/or reverse immune exhaustion caused by HIV infection while specifically targeting HIV-infected cells. To date, a single abstract has been presented, which suggests that exposure to PD-1 antibodies induces expression of HIV. However, the relevance and validity of this observation were weakened by the fact that the cells studied were obtained from viremic patients. ${ }^{19}$ A clinical trial in development to determine the effect of PD-1 antibodies on persistent infection in ART-treated patients is currently delayed by unanswered safety concerns arising from observed toxicities following the use of the drug in oncology trials (Table 1).

\section{Vaccination}

Several studies have reported increases in viral expression following routine vaccination against other pathogens in HIV-infected individuals, which may be attributable to mild activation of the immune system. ${ }^{20}$ However, the increased viral loads and T-cell activation after immunization were not associated with better viral control when ART was interrupted, ${ }^{20}$ indicating that this therapy may need to be performed in the context of ART or other therapeutics. In an interesting and provocative study, Persaud and colleagues ${ }^{21}$ immunized patients with a poxvirus vaccine engineered to express HIV antigens and observed a significant, albeit transient, decrease in replication-competent HIV in the resting T-cell reservoir (Table 1). These results suggest that such approaches might activate latent virus and/or induce an immune response to low-level antigen production in some cells, thereby resulting in a decline of the number of latently infected T cells. Such vaccines have 
been studied and well tolerated in HIV-infected patients, and so this strategy has potential for use in treating latent HIV. ${ }^{22}$

\section{Other potential targets}

Although HDACis are currently the most promising candidates for the "kick and kill" approach, many other cellular pathways are involved in the maintenance of latent HIV, and compounds targeting these pathways may enter clinical trials in the near future. Here, we briefly discuss examples of these pathways and a few of the promising compounds under study.

Activation of the protein kinase $\mathrm{C}(\mathrm{PKC})$ and nuclear factor- $\kappa \mathrm{B}(\mathrm{NF}-\kappa \mathrm{B})$ pathways induces expression of quiescent HIV proviruses. Once activated, PKC phosphorylates I $\mathrm{B} \mathrm{B}$, which results in the release of NF- $\kappa B$. NF- $\kappa B$ then translocates to the nucleus, where it binds to the HIV LTR and promotes transcriptional activation (Figure 1). Drugs targeting these pathways, such as tumor necrosis factor-a, prostratin, and bryostatin, are currently under study for the treatment of latent HIV infection. ${ }^{23-25}$ Prostratin, a phorbol ester that targets both the PKC and NF- $\kappa \mathrm{B}$ pathways, activates HIV transcription in several J-lat cell-line models of HIV latency. ${ }^{24}$ In addition, prostratin in combination with the HDACis VPA and VOR synergistically increases the amount of virus produced from cell-line models of HIV latency. ${ }^{26}$ Bryostatin, a macrocyclic lactone, is another candidate that targets the PKC and NF- $\kappa \mathrm{B}$ pathways. In addition to modulating the PKC and NF- $\kappa \mathrm{B}$ pathways, bryostatin-1 also downregulates the CD4 receptor on T cells, which limits the ability of HIV to infect these cells (Figure 1). ${ }^{25}$ Importantly, bryostatin does not activate T cells and has low cytotoxicity. ${ }^{27}$ Bryostatin has entered a phase II clinical trial for the treatment of ovarian cancer ${ }^{28}$ However, in this trial, despite a partial response of some patients to the treatment, severe myalgia developed in all study participants. ${ }^{28}$ Although studies to advance bryostatin for future treatment of latent HIV are planned, testing will have to be done cautiously in light of these side effects.

HMBA, a modulator of the HMBA-inducible 1 (HEXIM1) component of the 7SK small nuclear ribonucleoprotein complex, also stimulates HIV transcription. ${ }^{29,30}$ HMBA activates the PI3K/Akt pathway, resulting in HEXIM1 phosphorylation and release of the transcriptional elongation factor positive transcription elongation factor $b$ from the transcriptionally repressive 7SK small nuclear ribonucleoprotein complex/HEXIM complex. ${ }^{31}$ Following its release, positive transcription elongation factor $\mathrm{b}$ is recruited to HIV transactivation response element by HIV transactivator of transcription, which facilitates productive transcription elongation (Figure 1). HMBA reached phase II clinical trials for acute myelogenous leukemia, ${ }^{32}$ and, although the drug was tolerable in this setting, further studies have not been pursued owing to lack of efficacy in cancer. HMBA was also rapidly metabolized, eventually requiring continuous IV infusion in studies to achieve detectable drug levels. Clinical trials for treatment of latent HIV have not been pursued.

In addition to histone deacetylation and signaling cascades, other possible targets for therapy are being investigated. Among these are enzymes that methylate histone proteins or DNA. Depending on the site of methylation and the number of methyl groups present, these modifications can recruit proteins that activate or repress transcription. 5-Aza-2'deoxycytidine (5-Aza-CdR), an inhibitor of DNA methyltransferases, activates transcription from the HIV LTR in several cell-line models of latent HIV (Figure 1). ${ }^{33}$ Furthermore, several histone methyltransferases, including SUV39, G9a, and EZH2, have been demonstrated to be involved in transcriptional modification of HIV. ${ }^{34}$ Significantly, the histone methyltransferase inhibitors Chaetocin, BIX-01294, and DZNep reactivate HIV transcription in cell-line models of HIV latency. However, the relevance of these observations to clinical applications remains unproven and awaits the assessment of these 
compounds in authentic resting cells from patients. ${ }^{34}$ Furthermore, the clinical safety of these drugs has not been assessed outside of limited studies in advanced malignancies, and so the development and clinical validation of safe and effective histone methyltransferase inhibitors and DNA methyltransferase inhibitors remain important research goals.

Additional compounds that may relieve HIV latency are currently under investigation. Xing and colleagues ${ }^{35}$ conducted a drug library screen to identify compounds that activate HIV transcription in a primary cell model of HIV latency, and derivatives of quinolin-8-ol, a bivalent cation chelator, were identified (Figure 1). Micheva-Viteva and colleagues ${ }^{36}$ also performed a high-throughput screen of 200,000 compounds on a lymphoid cell-line model of HIV latency and found that AV6, a 4-3', 4'-dichloroanilino-6-methoxyquinoline compound, was able to induce transcription of HIV. Although the mechanisms of viral activation are not known for these newly identified compounds, they did not significantly activate $\mathrm{T}$ cells. Thus, these new compounds represent additional classes of drugs that show potential as a treatment for latent HIV infection. ${ }^{36}$

\section{Removal of proviral DNA}

The most straightforward solution to eliminating latent reservoirs is simply to remove integrated HIV genomes from infected cells. However, the technological hurdles to achieving this goal are enormous. This would require vectors that identify and act on a specific DNA sequence within the billions of bases of DNA in a cell, where the targets lie predominantly within resting cells that are difficult to transduce and within $<0.00001 \%$ of the total cell population in all host tissues. All this would have to be achieved with exquisite specificity to avoid inducing unacceptable damage to DNA host sequences. Toward this goal, HIV-specific recombinase enzymes have been evolved to recognize sequences within the HIV LTRs and to remove the intervening HIV DNA ${ }^{37}$, resulting in cells that no longer harbor a functional HIV genome (Figure 2). Clinically, this should result in a depletion of the viral reservoir in the patient following treatment. Although the technology is still evolving, encouraging results have been obtained, and additional studies have been performed to improve the therapy and discover more efficient ways to introduce the enzyme into cells. ${ }^{38}$ However, at present, this technology can benefit only infected cells that are specifically isolated and purged of proviral genomes; therefore, many infected cells will remain in the peripheral blood and other reservoirs.

\section{Elimination of cells expressing HIV proteins}

Therapeutics designed to purge latent reservoirs may have varying effectiveness among cells or cell types. The result of this may be full activation of latent, replication-competent provirus in some cells and minimal activation in others. In the first case, infectious viral particles that are capable of infecting bystander cells will be produced, increasing the risk of de novo infection events. In the second case, infected cells may escape detection by the immune system and return to latency. The use of HIV-dependent suicide genes addresses both of these problems. Anti-HIV suicide gene constructs encode a toxic or proapoptotic protein whose expression is controlled by the HIV LTR, which means that the toxin should only be expressed when HIV Tat is expressed (Figure 2). However, early suicide gene constructs either suffered from nonspecific killing of uninfected cells due to basal transcription off of the HIV promoter or conversely failed to kill infected cells rapidly or efficiently enough due to suboptimal suicide gene construction. ${ }^{39}$ Recent constructs have been improved through inclusion of elements that make expression dependent not only on HIV Tat (HIV LTR) but also on Rev (Rev response element, HIV inhibitory sequences), which increases specificity, and efficacy has been improved through inclusion of more potent proapoptotic genes (Figure 2) ${ }^{39}$ However, because expression of the suicide gene and subsequent cell death require some level of Tat and Rev expression, this approach by 
itself will be ineffective at purging latent provirus. However, in combination with antilatency therapeutics, such as the small-molecule drugs addressed earlier in this review, suicide genes could serve as an adjunctive therapy. In this role, suicide genes can be used to destroy infected cells prior to virion release, thus reducing viremia and the likelihood of additional infection events. Furthermore, they may allow destruction of infected cells undergoing low-level proviral induction that is sufficiently strong to trigger expression of the toxic proteins but insufficient to alert the immune system to the presence of the provirus.

\section{Introduction to a functional cure}

A functional cure either suppresses HIV replication or creates an immune system that can control HIV infection in the presence of active HIV replication. Although replicationcompetent HIV would still be present in this scenario, patients would be able to discontinue ART without adverse effects. Many HIV gene therapy-based strategies attempt to control HIV by supporting immune function, often through the establishment and expansion of HIV-resistant cells. One way of attempting this is by inhibiting the expression or activity of cellular proteins that are required for viral entry, such as CCR5 and CXCR4, the coreceptors for R5- and X4-tropic viruses, respectively. Importantly, both CCR5 (refs. 40,41) and CXCR4 (refs. 42,43) levels can be reduced or eliminated in T cells. Strategies to create such cells include direct modification of coreceptor gene sequences and RNA-based destruction or translational silencing of coreceptor messenger RNA (mRNA). Another approach being tested as a way to support immune function is to reverse the immune exhaustion that occurs during chronic viral infections and to allow the revitalized immune system to control the HIV infection and any secondary infections.

\section{Creating HIV-resistant cells}

HIV binds to CD4 and uses either the CCR5 or CXCR4 coreceptors to facilitate entry into cells. CCR5 is the predominant coreceptor used by HIV during both initial infection and subsequently. ${ }^{44,45}$ Viruses using the CXCR4 coreceptor are more often seen as the disease progresses. ${ }^{46,47}$ Of clinical significance, individuals who are homozygous for the naturally occurring $c c r 5 \Delta 32$ mutation are resistant to CCR5-tropic HIV infection, ${ }^{40,48}$ whereas some heterozygous individuals exhibit delayed HIV-disease progression. ${ }^{49}$ The definitive proof that the homozygous mutation in CCR5 can contribute to an HIV cure is the experience of the "Berlin patient." This HIV-infected patient developed myelogenous leukemia and received a hematopoietic stem cell transplant using cells from a ccr5 32 -homozygous donor; after more than 7 years without ART, his HIV viral load remains undetectable. ${ }^{50,51}$ Because this patient experienced a number of procedures and conditions associated with the treatment, including radiation therapy, chemotherapy, anti-T-cell immunoglobulin treatment, and graft-vs.-host disease, it is unclear exactly which components of his treatment and clinical course contributed to his cure.

Nevertheless, the results of this case stimulated the field to reconsider the possibility of eradication of HIV infection, and other groups are currently attempting to repeat the experience of a transplant-based cure for HIV infection. In one study conducted by Henrich and colleagues, ${ }^{52}$ two patients who underwent an allogeneic stem cell transplant with homozygous $c c r 5 \Delta 32$ donor cells had undetectable levels of HIV in their peripheral blood, as determined by PCR for HIV DNA and an assay for HIV RNA that detects as little as one copy per milliliter, between 8 and 17 months after the allogeneic stem cell transplant. Further studies are currently being conducted to determine the duration of this effect and to assess whether HIV is detectable in additional viral reservoirs. However, the limited availability of $c c r 5 \Delta 32$-homozygous donors and the risks associated with the irradiation and chemotherapy that comprise part of the treatment probably make allogeneic hematopoietic 
stem cell transplant a tool to study clearance of HIV infection, but it does not serve as a practical solution for HIV patients.

An alternative to donor hematopoietic stem cell transplants is to engineer a CCR5 deletion within a patient's own cells and then perform an autologous transplant. Using specialized enzymes containing both DNA-recognition and endonucleolytic domains, it is possible to introduce these deletions by creating lesions at specific sites within the gene. These enzymes, known as zinc-finger nucleases (ZFNs), bind to the DNA through sequencespecific interactions between their zinc-finger domains and the DNA (Figure 3). Following binding, the ZFN cleaves the gene, creating double-strand breaks that are recognized by the cell's DNA repair machinery and repaired primarily by nonhomologous end joining (Figure 3 ). This type of repair frequently creates an insertion or deletion in the gene, and these mutations often result in translation of a nonfunctional protein.

ZFNs have been used to manipulate CCR5 expression in $\mathrm{CD}^{+} \mathrm{T}$ cells and precursor cells. A CCR5-specific ZFN introduced into cultured $\mathrm{CD}^{+} \mathrm{T}$ cells results in stable protection against HIV infection that can be passed on to progeny cells. ${ }^{53}$ Furthermore, introducing these cells into HIV-infected mice leads to reduced viral load and higher $\mathrm{CD}^{+} \mathrm{T}$ cell levels as compared with those in control mice. ${ }^{53}$ Similar results are achieved by introducing CCR5-ZFNs into CD34+ hematopoietic stem/progenitor cells. ${ }^{54}$ When these cells are engrafted into HIV-infected mice, there is rapid selection for CCR5-null cells, resulting in lower viral load and higher levels of $\mathrm{CD} 4^{+} \mathrm{T}$ cells as compared with those in control mice. ${ }^{54}$

Ongoing clinical trials (NCT00842634, NCT01044654, and NCT01252641) are addressing the safety and efficacy of CCR5-ZFN-treated autologous cells, known as SB-728-T, in humans, including effects on $\mathrm{CD} 4^{+} \mathrm{T}$-cell counts, viral load, and the ability of the cells to localize to anatomical reservoirs (Table 1). Preliminary results indicate that treatment is generally well tolerated and leads to increased $\mathrm{CD} 4{ }^{+} \mathrm{T}$-cell levels. ${ }^{55,56}$ Significantly, SB-728-T cells not only persist over time but also appear to expand and undergo trafficking to the gut-associated lymphoid tissue. These results suggest that a small initial pool of HIVresistant cells may persist after transplantation. However, whether these cells exhibit normal immune function and can control HIV replication over time remains to be demonstrated.

Despite these encouraging results, inhibiting CCR5 expression does not protect against CXCR4-tropic viruses and may drive selection for either X4-specific or dual-tropic HIV viruses. ${ }^{57,58}$ One possible solution is to inhibit both the CCR5 and CXCR4 coreceptors simultaneously in T cells, thereby preventing viral entry regardless of tropism. ZFNs targeting CXCR4 effectively reduce CXCR4 levels and convey long-term resistance to X4tropic HIV infection in cell lines and humanized mice.$^{59,60}$ Not unexpectedly, resistance in mice is limited by selection for R5-tropic viruses ${ }^{59}$ However, when homozygous CCR5 32 $\mathrm{CD}^{+}{ }^{+} \mathrm{T}$ cells were treated with CXCR4 ZFNs, the cells became resistant to both R5- and $\mathrm{X} 4$-tropic HIV,${ }^{59}$ supporting the notion that simultaneous reduction of the expression of both coreceptors in patient-derived cells is a therapeutic possibility. If so, these cells could be used as a founder population of HIV-resistant cells to help re-establish or preserve immune function and prevent disease progression. However, whereas the effects of loss of CCR5 on immune function appear modest, the effects of the deletion of CXCR4 on immune function are less well understood, and the effects of simultaneous loss of both receptors remain unexplored.

\section{Modification of hematopoietic progenitor cells with RNA-based therapeutics}

Many RNA-based tools, such as ribozyme biochemistry and RNA interference (RNAi), are being investigated for their potential application to treat HIV infection. Ribozymes are catalytic RNA molecules that do not require proteinaceous partners for their activity and can 
be evolved in the laboratory to cleave a desired sequence. RNAi involves the sequencespecific targeting of an mRNA by a small RNA molecule, resulting in translational silencing or destruction of the mRNA and subsequent depletion of the target protein.

A handful of recent studies have addressed RNA-based therapeutics for treating HIV infection. Most have targeted viral mRNAs by RNAi with the hope of holding viral replication in check, although there has also been interest in targeting cellular factors such as CCR5. Many of these studies are in the preclinical phase and have focused on one or more aspects of a therapy known as Triple-R, which combines expression of an anti-CCR5 ribozyme, anti-Tat and Rev short hairpin RNAs, and a transactivation response element decoy RNA containing a nucleolar localization tag (Figure 3) ${ }^{61}$ Results from in vitro and mouse model studies reveal that this type of therapy is effective at protecting cells from HIV infection. However, in a phase I clinical trial, the outcome was not as encouraging. ${ }^{61}$ Although the therapeutic RNAs were detectable in some patients over time and the therapy was generally well tolerated, the effect of Triple-R on HIV infection was negligible (Table 1). ${ }^{61}$ However, this may be less a reflection on RNA-based therapy as a treatment strategy and more an indication of problems with clinical protocols, especially the general challenges that vex gene therapy approaches-vector delivery and functional persistence.

\section{Reversal of immune exhaustion}

The pathogen-specific immune response to HIV is determined by the T-cell receptor major histocompatibility complex-peptide complexes formed during antigen presentation and subsequent binding of positive or negative costimulatory molecules to their specific receptors or ligands. Despite clinically successful ART, viral antigenemia persists at low levels, causing $\mathrm{T}$ cells to gradually lose their effector functions, including cytotoxicity and cytokine secretion, as well as their proliferative capacity. ${ }^{62,63}$ Progressive functional impairment of T cells, called immune exhaustion, is a hallmark of HIV infection and appears to be multifactorial. ${ }^{64}$ Inhibitory receptors involved in T-cell exhaustion are potential targets for immunotherapeutic strategies that, if effective, could reverse immune exhaustion and allow patients to control HIV without ART. Among these, PD-1 is among the best studied and the one most likely to be relevant for near-term immunotherapy. Figure 3 shows a schematic representation of the receptors involved in immune responses and details the use of anti-PD-1 antibodies as a potential therapy that may reverse immune exhaustion during chronic HIV infection.

Barber and colleagues ${ }^{65}$ demonstrated the potential application of anti-PD-1 antibodies for HIV therapy when they observed improved T-cell function after blocking the PD-1 receptor in mice. Subsequently, two studies demonstrated restoration of CD4- and CD8-specific responses and cytokine production in vitro upon blocking PD-1. ${ }^{66,67}$ Furthermore, Petrovas and colleagues ${ }^{68}$ demonstrated that cytotoxic T lymphocytes expressing PD-1 were more susceptible to apoptosis, although it remains unclear whether blocking PD-1 improves T-cell survival. In vivo blockade of PD-1 in chronically simian immunodeficiency virus-infected rhesus macaques resulted in no changes in SIV-specific T-cell function or numbers after a single injection of PD-1 antibody, ${ }^{69}$ whereas enhanced SIV-specific immune responses were observed after four repeated injections. ${ }^{70}$ Clinical trials for hematological malignancies using antibodies to block the interaction between the PD-1 receptor and its ligand (PD-L1) have recently been reported, with administration of a humanized anti-PD-1 antibody resulting in a sustained increase in $\mathrm{CD} 4^{+} \mathrm{T}$-cell counts after a single dose $\mathrm{e}^{71}$ and restoration of T-cell responses. ${ }^{71,72}$ Furthermore, there have been encouraging results from the use of an anti-PD-1 antibody as an adjuvant in lentiviral vaccines to enhance antigen-specific immune responses. ${ }^{73}$ However, concerns about using PD-1 blockade as a therapeutic 
approach for HIV infection include the possibility that enhancing antiviral responses by blocking PD-1 could also enhance activation in an already preactivated system.

Despite the high expression of PD-1 in exhausted T cells during HIV infection, its heterogeneous expression in distinct cellular subsets and tissues and the observation that blockade of PD-1 results in only partial restoration of T-cell function ${ }^{74}$ suggest a complex system in which additional inhibitory mediators may interact. In support of this, it was reported that simultaneous blockade of PD-1 and another inhibitory molecule in mice improved viral control and cytotoxic-T-lymphocyte function better than blocking PD-1 alone. ${ }^{75}$ Furthermore, because immune exhaustion is gradual, inhibitory receptors accumulate over time on T-cell membranes, and this correlates with HIV viral load better than expression of a single molecule. ${ }^{75}$ Therefore, inhibition of PD-1 may need to be combined with additional therapies for patients to regain full $\mathrm{T}$-cell function.

\section{CHALLENGES}

Despite the many promising inroads toward a cure for HIV, many challenges exist. Among these is a pressing need for more refined cellular models of HIV latency and standardization of how active and latent HIV is detected. Surmounting these challenges will help streamline the avenue toward a cure.

\section{Cellular models of latency}

Many cell-based models of HIV latency have been developed, including cell-line and primary-cell models. Although cell-line models offer a preliminary platform for drug discovery and characterization, they have characteristics that make them less attractive as standard latency models. First, cell lines are often immortalized and clonal, meaning that the viral integration site is the same in every cell. Because immortalizing a cell can affect its biology, and because the mechanisms of latency can vary depending on where the provirus is located in the host genome, cell lines are not deemed completely representative of latency. In addition, experimental results often vary from one cell line to the next.

Primary cells isolated from HIV-negative donors are not immortalized or clonal and, when infected and cultured ex vivo, are generally assumed to be superior to cell lines as models of latency. Currently, there are several primary cell models of latency, and although each tends to model one or more aspects of latency effectively, experimental results derived from these models do not completely mirror those obtained from patient cells. Therefore, the search for a standard and representative model of latency continues. This may be a Sisyphean task, given that the biological diversity of latency may be sufficient to thwart the development of any single, fully representative model. In the meantime, researchers are working to define a minimal panel of existing primary cell models that can be used in tandem to give a more complete representation of the patient milieu and facilitate drug characterization. Although this is a less than perfect solution, it will be a useful advance for the field.

\section{Detecting HIV}

There is as yet no single, universally employed, standardized method for measuring latent HIV infection that is rapid, accurate, sensitive, and economical. Instead, a diversity of methods are used, including PCR- and protein-based assays. Each method provides valuable information, but because the assays measure disparate aspects of expression and often exhibit variability from lab to lab, rectifying study results can be challenging. This presents a significant technical roadblock to efficient characterization of therapeutics and hinders our understanding of their precise effects on latency. 
PCR-based assays are widely popular and can be used to measure HIV expression at different stages of the virus life cycle that other methods cannot. Being increasingly sensitive, rapid, and economical, these assays fit several criteria that are required for a standardized assay. Quantitative real-time PCR can measure nonintegrated 2-LTR episomes that are formed by nonhomologous end joining of viral DNA; integrated HIV DNA, which is required for later stages of virus expression; HIV mRNA, indicative of proviral transcription; and cell-associated or cell-free genomic RNA. However, PCR-based methods not only allow but often require measurement of several different aspects of viral replication to ensure that accurate conclusions are drawn. Furthermore, artifacts can be quite common, making clever experimental design and the use of adequate controls especially critical. Of note, PCR-based methods do not directly measure replication-competent virus, which introduces an additional level of uncertainty to these assays.

The current gold-standard method for detecting and quantifying HIV replication is the viral outgrowth assay. However, its widespread use is hindered because the assay is resourceintensive and time-consuming. One of the greatest challenges is the requirement for patientderived cells, which are usually unavailable to researchers unaffiliated with a clinical facility. Moreover, the assay requires $\sim 3$ weeks to complete, making it far from rapid. Despite these challenges, the viral outgrowth assay, which measures p24 ${ }^{\text {gag }}$ levels by enzyme-linked immunosorbent assay as an end point, is the most accurate and sensitive measure of HIV replication available. At present, no other assay seems poised to unseat the viral outgrowth assay as the gold standard, but much work is focused on this goal.

\section{Conclusion}

Although HIV infection is generally manageable with lifelong HAART (highly active ART), there is a continuing investment of effort and resources aimed at the development of an effective and economical treatment strategy to address HIV latency. Regardless of which therapies are successful, an important consideration is the ability to deploy treatment to millions of people around the world in a way that is logistically and economically feasible. Cell transplantation is not feasible, short of dramatic and unimagined advances in technology. Ultimately, it seems likely that the most useful treatments will be ones that can be administered directly to patients without the need for sophisticated equipment or techniques and that can be delivered specifically to the necessary cell types with minimal toxicity. One or more of the therapy options presented in this review may one day fit these requirements. Currently, the most promising approach that could possibly be scaled up for treatment of people in resource-limited environments is the concept of "kick and kill." However, if the delivery of gene therapy-based approaches is optimized, it would also be widely distributable to large numbers of people all over the world. The current requirement for immune exhaustion reversal therapies to be administered repeatedly makes them the least likely to be a worldwide solution for treatment of latent HIV. In the meantime, the search continues for more directed and effective approaches for eliminating HIV latency.

In conclusion, significant advances are being made in the development of therapies for the treatment of latent HIV. Currently, small molecules that reactivate HIV from the latent state are the furthest along in terms of the diversity of options and the advancement to clinical trials. However, gene therapy-based and immune-based strategies are developing at a fast pace and may begin to enter clinical trials in the near future. The development of several collaborative groups that include experts from many different fields has led to the development of several novel approaches and has significantly increased the pace of discovery in the field. The next step for many of the approaches discussed above is to optimize them to the point that they can be tested in clinical trials. Once the effectiveness and safety of these different approaches has been assessed in clinical trials, it will be 
important to determine whether a single method or a combination of these methods will be the most effective and easily distributable way to treat latent HIV infection.

\section{Acknowledgments}

D.M.M. has received research materials or funding or consulting honoraria from Merck, BMS, GSK, Argos, Tibotec, Gilead, Chimerix, Boehringer, and Viiv. He is coinventor on a patent for the use of vorinostat in HIV infection but has remanded ownership of the patent to the University of North Carolina.

\section{References}

1. WHO, UNICEF, and UNAIDS. Progress Report 2011: Global HIV/AIDS Response. 2011. <http:// www.who.int/hiv/pub/progress_report2011/en/index.html>

2. Colin L, Van Lint C. Molecular control of HIV-1 postintegration latency: implications for the development of new therapeutic strategies. Retrovirology. 2009; 6:111. [PubMed: 19961595]

3. Van Lint C, Emiliani S, Ott M, Verdin E. Transcriptional activation and chromatin remodeling of the HIV-1 promoter in response to histone acetylation. EMBO J. 1996; 15:1112-1120. [PubMed: 8605881]

4. Keedy KS, Archin NM, Gates AT, Espeseth A, Hazuda DJ, Margolis DM. A limited group of class I histone deacetylases acts to repress human immunodeficiency virus type 1 expression. J. Virol. 2009; 83:4749-4756. [PubMed: 19279091]

5. Krishnan V, Zeichner SL. Host cell gene expression during human immunodeficiency virus type 1 latency and reactivation and effects of targeting genes that are differentially expressed in viral latency. J. Virol. 2004; 78:9458-9473. [PubMed: 15308739]

6. Lehrman G, et al. Depletion of latent HIV-1 infection in vivo: a proof-of-concept study. Lancet. 2005; 366:549-555. [PubMed: 16099290]

7. Archin NM, et al. Administration of vorinostat disrupts HIV-1 latency in patients on antiretroviral therapy. Nature. 2012; 487:482-485. [PubMed: 22837004]

8. Siliciano JD, et al. Stability of the latent reservoir for HIV-1 in patients receiving valproic acid. J. Infect. Dis. 2007; 195:833-836. [PubMed: 17299713]

9. Matalon S, Rasmussen TA, Dinarello CA. Histone deacetylase inhibitors for purging HIV-1 from the latent reservoir. Mol. Med. 2011; 17:466-472. [PubMed: 21424110]

10. Lewin, SR. HIV latency and eradication: clinical perspectives. 19th Conference on Retroviruses and Opportunistic Infections; 5-8 March 2012; Seattle, WA.

11. Wightman F, Ellenberg P, Churchill M, Lewin SR. HDAC inhibitors in HIV. Immunol. Cell Biol. 2012; 90:47-54. [PubMed: 22083528]

12. Vojinovic J, et al. Safety and efficacy of an oral histone deacetylase inhibitor in systemic-onset juvenile idiopathic arthritis. Arthritis Rheum. 2011; 63:1452-1458. [PubMed: 21538322]

13. Furlan A, et al. Pharmacokinetics, safety and inducible cytokine responses during a phase 1 trial of the oral histone deacetylase inhibitor ITF2357 (givinostat). Mol. Med. 2011; 17:353-362. [PubMed: 21365126]

14. Leoni $\mathrm{F}$, et al. The antitumor histone deacetylase inhibitor suberoylanilide hydroxamic acid exhibits antiinflammatory properties via suppression of cytokines. Proc. Natl. Acad. Sci. USA. 2002; 99:2995-3000. [PubMed: 11867742]

15. Archin NM, Espeseth A, Parker D, Cheema M, Hazuda D, Margolis DM. Expression of latent HIV induced by the potent HDAC inhibitor suberoylanilide hydroxamic acid. AIDS Res. Hum. Retroviruses. 2009; 25:207-212. [PubMed: 19239360]

16. Xing S, et al. Disulfiram reactivates latent HIV-1 in a Bcl-2-transduced primary CD4+ T cell model without inducing global T cell activation. J. Virol. 2011; 85:6060-6064. [PubMed: 21471244]

17. Doyon G, Zerbato J, Mellors JW, Sluis-Cremer N. Disulfiram reactivates latent HIV-1 expression through depletion of the phosphatase and tensin homolog (PTEN). AIDS. 2012 e-pub ahead of print 27 June 2012. 
18. Spivak, A., et al. Safety and feasibility of using disulfiram to enhance HIV transcription among long-term ARV-treated adults: preliminary results from a pilot study. 19th Conference on Retroviruses and Opportunistic Infections; 5-8 March 2012; Seattle, WA.

19. Da Fonseca, S., et al. Purging the HIV-1 reservoir through disruption of the PD-1 pathway. 18th Conference on Retroviruses and Opportunistic Infections; 27 February-2 March 2011; Boston, MA.

20. Castro $P$, et al. Influence of a vaccination schedule on viral load rebound and immune responses in successfully treated HIV-infected patients. AIDS Res. Hum. Retroviruses. 2009; 25:1249-1259. [PubMed: 19943787]

21. Persaud D, et al. Effect of therapeutic HIV recombinant poxvirus vaccines on the size of the resting CD4+ T-cell latent HIV reservoir. AIDS. 2011; 25:2227-2234. [PubMed: 21918423]

22. García F, León A, Gatell JM, Plana M, Gallart T. Therapeutic vaccines against HIV infection. Hum. Vaccin. Immunother. 2012; 8:569-581. [PubMed: 22634436]

23. Osborn L, Kunkel S, Nabel GJ. Tumor necrosis factor alpha and interleukin 1 stimulate the human immunodeficiency virus enhancer by activation of the nuclear factor kappa B. Proc. Natl. Acad. Sci. USA. 1989; 86:2336-2340. [PubMed: 2494664]

24. Williams SA, et al. Prostratin antagonizes HIV latency by activating NF-kappaB. J. Biol. Chem. 2004; 279:42008-42017. [PubMed: 15284245]

25. Boto WM, Brown L, Chrest J, Adler WH. Distinct modulatory effects of bryostatin 1 and staurosporine on the biosynthesis and expression of the HIV receptor protein (CD4) by T cells. Cell Regul. 1991; 2:95-103. [PubMed: 1863603]

26. Reuse S, et al. Synergistic activation of HIV-1 expression by deacetylase inhibitors and prostratin: implications for treatment of latent infection. PLoS ONE. 2009; 4:e6093. [PubMed: 19564922]

27. Mehla R, et al. Bryostatin modulates latent HIV-1 infection via PKC and AMPK signaling but inhibits acute infection in a receptor independent manner. PLoS ONE. 2010; 5:e11160. [PubMed: 20585398]

28. Morgan RJ Jr, et al. Phase II trial of bryostatin-1 in combination with cisplatin in patients with recurrent or persistent epithelial ovarian cancer: a California cancer consortium study. Invest. New Drugs. 2012; 30:723-728. [PubMed: 20936324]

29. Choudhary SK, Archin NM, Margolis DM. Hexamethylbisacetamide and disruption of human immunodeficiency virus type 1 latency in CD4(+) T cells. J. Infect. Dis. 2008; 197:1162-1170. [PubMed: 18419522]

30. Klichko V, Archin N, Kaur R, Lehrman G, Margolis D. Hexamethylbisacetamide remodels the human immunodeficiency virus type-1 (HIV-1) promoter and induces Tat-independent HIV-1 expression but blunts cell activation. J. Virol. 2006; 80:4570-4579. [PubMed: 16611917]

31. Contreras X, Barboric M, Lenasi T, Peterlin BM. HMBA releases P-TEFb from HEXIM1 and 7SK snRNA via PI3K/Akt and activates HIV transcription. PLoS Pathog. 2007; 3:1459-1469. [PubMed: 17937499]

32. Andreeff M, et al. Hexamethylene bisacetamide in myelodysplastic syndrome and acute myelogenous leukemia: a phase II clinical trial with a differentiation-inducing agent. Blood. 1992; 80:2604-2609. [PubMed: 1421378]

33. Fernandez G, Zeichner SL. Cell line-dependent variability in HIV activation employing DNMT inhibitors. Virol. J. 2010; 7:266. [PubMed: 20942961]

34. Friedman J, et al. Epigenetic silencing of HIV-1 by the histone H3 lysine 27 methyltransferase enhancer of Zeste 2. J. Virol. 2011; 85:9078-9089. [PubMed: 21715480]

35. Xing S, et al. Novel structurally related compounds reactivate latent HIV-1 in a bcl-2-transduced primary CD4+ T cell model without inducing global T cell activation. J. Antimicrob. Chemother. 2012; 67:398-403. [PubMed: 22160146]

36. Micheva-Viteva S, et al. High-throughput screening uncovers a compound that activates latent HIV-1 and acts cooperatively with a histone deacetylase (HDAC) inhibitor. J. Biol. Chem. 2011; 286:21083-21091. [PubMed: 21498519]

37. Sarkar I, Hauber I, Hauber J, Buchholz F. HIV-1 proviral DNA excision using an evolved recombinase. Science. 2007; 316:1912-1915. [PubMed: 17600219] 
38. Mariyanna L, et al. Excision of HIV-1 proviral DNA by recombinant cell permeable trerecombinase. PLoS ONE. 2012; 7:e31576. [PubMed: 22348110]

39. Huelsmann PM, et al. A suicide gene approach using the human pro-apoptotic protein tBid inhibits HIV-1 replication. BMC Biotechnol. 2011; 11:4. [PubMed: 21223573]

40. Samson M, et al. Resistance to HIV-1 infection in caucasian individuals bearing mutant alleles of the CCR-5 chemokine receptor gene. Nature. 1996; 382:722-725. [PubMed: 8751444]

41. Hutter G, Neumann M, Nowak D, Klein S, Kluter H, Hofmann WK. The effect of the CCR5delta32 deletion on global gene expression considering immune response and inflammation. J. Inflamm. (Lond). 2011; 8:29. [PubMed: 22029606]

42. Anderson J, Akkina R. CXCR4 and CCR5 shRNA transgenic CD34+ cell derived macrophages are functionally normal and resist HIV-1 infection. Retrovirology. 2005; 2:53. [PubMed: 16109172]

43. Ma Q, et al. Impaired B-lymphopoiesis, myelopoiesis, and derailed cerebellar neuron migration in CXCR4- and SDF-1-deficient mice. Proc. Natl. Acad. Sci. USA. 1998; 95:9448-9453. [PubMed: 9689100]

44. Schuitemaker $\mathrm{H}$, et al. Biological phenotype of human immunodeficiency virus type 1 clones at different stages of infection: progression of disease is associated with a shift from monocytotropic to T-cell-tropic virus population. J. Virol. 1992; 66:1354-1360. [PubMed: 1738194]

45. Keele BF, et al. Identification and characterization of transmitted and early founder virus envelopes in primary HIV-1 infection. Proc. Natl. Acad. Sci. USA. 2008; 105:7552-7557. [PubMed: 18490657]

46. Connor RI, Sheridan KE, Ceradini D, Choe S, Landau NR. Change in coreceptor use correlates with disease progression in HIV-1-infected individuals. J. Exp. Med. 1997; 185:621-628. [PubMed: 9034141]

47. Huang Y, et al. The role of a mutant CCR5 allele in HIV-1 transmission and disease progression. Nat. Med. 1996; 2:1240-1243. [PubMed: 8898752]

48. Liu R, et al. Homozygous defect in HIV-1 coreceptor accounts for resistance of some multiplyexposed individuals to HIV-1 infection. Cell. 1996; 86:367-377. [PubMed: 8756719]

49. de Roda Husman AM, et al. Association between CCR5 genotype and the clinical course of HIV-1 infection. Ann. Intern. Med. 1997; 127:882-890. [PubMed: 9382366]

50. Allers K, et al. Evidence for the cure of HIV infection by CCR5 $32 / \Delta 32$ stem cell transplantation. Blood. 2011; 117:2791-2799. [PubMed: 21148083]

51. Hutter G, et al. Long-term control of HIV by CCR5 Delta32/Delta32 stem-cell transplantation. N. Engl. J. Med. 2009; 360:692-698. [PubMed: 19213682]

52. Henrich, TJ., et al. Long-term reduction in peripheral blood HIV-1 reservoirs following reducedintensity conditioning allogeneic stem cell transplantation in two HIV-positive individuals. XIX International AIDS Conference; 22-27 July 2012; Washington, DC.

53. Perez EE, et al. Establishment of HIV-1 resistance in CD4+ T cells by genome editing using zincfinger nucleases. Nat. Biotechnol. 2008; 26:808-816. [PubMed: 18587387]

54. Holt N, et al. Human hematopoietic stem/progenitor cells modified by zincfinger nucleases targeted to CCR5 control HIV-1 in vivo. Nat. Biotechnol. 2010; 28:839-847. [PubMed: 20601939]

55. June, C., et al. Introduction of acquired CCR5 deficiency with zinc finger nuclease-modified autologous CD4 T cells (SB-728-T) correlates with increases in CD4 count and effects on viral load in HIV-infected subjects. 19th Conference on Retroviruses and Opportunistic Infections; 5-8 March 2012; Seattle, WA.

56. Lalezari, J., et al. A single infusion of zinc finger nuclease CCR5 modified autologous CD4 T cells (SB-728-T) increases CD4 counts and leads to decrease in HIV proviral load in an aviremic HIVinfected subject. 19th Conference on Retroviruses and Opportunistic Infections; 5-8 March 2012; Seattle, WA.

57. Westby M, et al. Emergence of CXCR4-using human immunodeficiency virus type 1 (HIV-1) variants in a minority of HIV-1-infected patients following treatment with the CCR5 antagonist maraviroc is from a pretreatment CXCR4-using virus reservoir. J. Virol. 2006; 80:4909-4920. [PubMed: 16641282] 
58. Nedellec R, Coetzer M, Lederman MM, Offord RE, Hartley O, Mosier DE. Resistance to the CCR5 inhibitor 5P12-RANTES requires a difficult evolution from CCR5 to CXCR4 coreceptor use. PLoS ONE. 2011; 6:e22020. [PubMed: 21760945]

59. Wilen CB, et al. Engineering HIV-resistant human CD4+ T cells with CXCR4-specific zinc-finger nucleases. PLoS Pathog. 2011; 7:e1002020. [PubMed: 21533216]

60. Yuan J, et al. Zinc-finger nuclease editing of human CXCR4 promotes HIV-1 CD4(+) T cell resistance and enrichment. Mol. Ther. 2012; 20:849-859. [PubMed: 22273578]

61. DiGiusto DL, et al. RNA-based gene therapy for HIV with lentiviral vector-modified CD34(+) cells in patients undergoing transplantation for AIDS-related lymphoma. Sci. Transl. Med. 2010; 2:36ra43.

62. Wherry EJ. T cell exhaustion. Nat. Immunol. 2011; 12:492-499. [PubMed: 21739672]

63. Palmer S, et al. Low-level viremia persists for at least 7 years in patients on suppressive antiretroviral therapy. Proc. Natl. Acad. Sci. USA. 2008; 105:3879-3884. [PubMed: 18332425]

64. Migueles SA, et al. Defective human immunodeficiency virus-specific CD8+ T-cell polyfunctionality, proliferation, and cytotoxicity are not restored by antiretroviral therapy. J. Virol. 2009; 83:11876-11889. [PubMed: 19726501]

65. Barber DL, et al. Restoring function in exhausted CD8 T cells during chronic viral infection. Nature. 2006; 439:682-687. [PubMed: 16382236]

66. Day CL, et al. PD-1 expression on HIV-specific T cells is associated with T-cell exhaustion and disease progression. Nature. 2006; 443:350-354. [PubMed: 16921384]

67. Trautmann L, et al. Upregulation of PD-1 expression on HIV-specific CD8+ T cells leads to reversible immune dysfunction. Nat. Med. 2006; 12:1198-1202. [PubMed: 16917489]

68. Petrovas C, et al. SIV-specific CD8+ T cells express high levels of PD1 and cytokines but have impaired proliferative capacity in acute and chronic SIVmac251 infection. Blood. 2007; 110:928936. [PubMed: 17440051]

69. Finnefrock AC, et al. PD-1 blockade in rhesus macaques: impact on chronic infection and prophylactic vaccination. J. Immunol. 2009; 182:980-987. [PubMed: 19124741]

70. Velu V, et al. Enhancing SIV-specific immunity in vivo by PD-1 blockade. Nature. 2009; 458:206-210. [PubMed: 19078956]

71. Berger R, et al. Phase I safety and pharmacokinetic study of CT-011, a humanized antibody interacting with PD-1, in patients with advanced hematologic malignancies. Clin. Cancer Res. 2008; 14:3044-3051. [PubMed: 18483370]

72. Rosenblatt J, et al. PD-1 blockade by CT-011, anti-PD-1 antibody, enhances ex vivo T-cell responses to autologous dendritic cell/myeloma fusion vaccine. J. Immunother. 2011; 34:409-418. [PubMed: 21577144]

73. Dai B, Xiao L, Bryson PD, Fang J, Wang P. PD-1/PD-L1 blockade can enhance HIV-1 Gagspecific t cell immunity elicited by dendritic cell-directed lentiviral vaccines. Mol. Ther. 2012; 20:1800-1809. [PubMed: 22588271]

74. Jin HT, et al. Cooperation of Tim-3 and PD-1 in CD8 T-cell exhaustion during chronic viral infection. Proc. Natl. Acad. Sci. USA. 2010; 107:14733-14738. [PubMed: 20679213]

75. Kassu A, et al. Regulation of virus-specific CD4+ T cell function by multiple costimulatory receptors during chronic HIV infection. J. Immunol. 2010; 185:3007-3018. [PubMed: 20656923] 


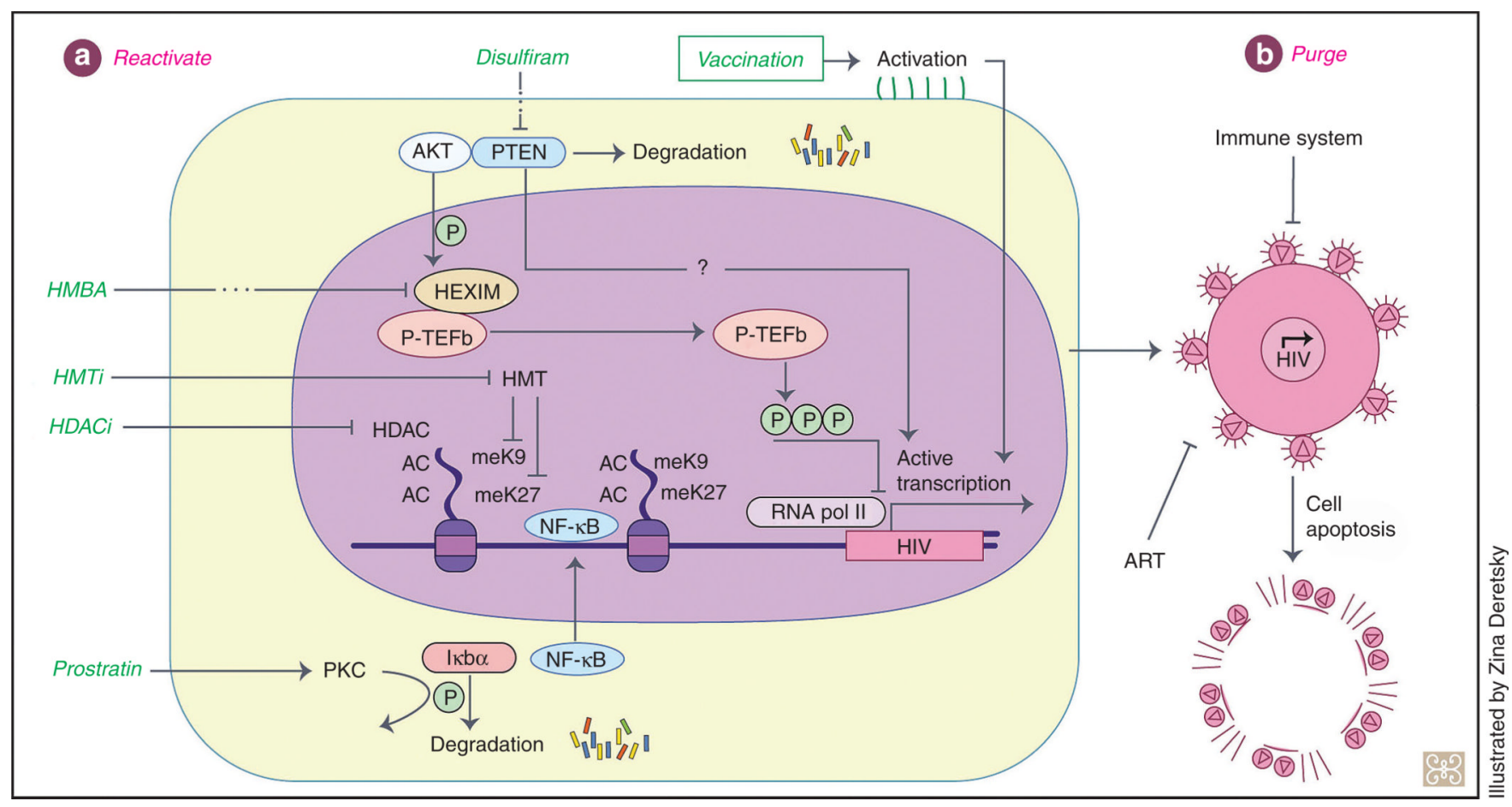

Figure 1.

Induction and clearance strategies. (a) Induction. Several small molecules have been identified that are able to induce transcription of quiescent HIV proviruses. Prostratin, tumor necrosis factor- $\mathrm{a}$, and bryostatin have been demonstrated to act on the protein kinase $\mathrm{C}$ / nuclear factor $\kappa \mathrm{B}(\mathrm{PKC} / \mathrm{NF}-\kappa \mathrm{B})$ pathway. Activated PKC phosphorylates $\mathrm{I} \kappa \mathrm{B} a$, causing release of $N F-\kappa B$. NF- $\kappa B$ then translocates to the nucleus, where it binds to the promoter of HIV and promotes transcription. Histone deacetylase (HDAC) inhibitors directly inhibit deacetylation of histones at the HIV long terminal repeat (LTR), which is associated with induction of transcription. Similarly, histone methyltransferase inhibitors (HMTi) inhibit the methylation of histone tails, which are associated with transcription from the HIV promoter in some model systems. Hexamethylene bisacetamide (HMBA) causes activation of the Akt pathway, which may result in phosphorylation of HMBA inducible 1 (HEXIM). This may result in the release of positive transcription elongation factor $b(\mathrm{p}-\mathrm{TEFb})$ from the inhibitory HEXIM complex, allowing p-TEFb access to the HIV LTR, where it phosphorylates RNA pol II and primes the LTR for transcription. Disulfiram has been shown to act upstream of HMBA, perhaps by causing degradation of phosphatase and tensin homolog, an inhibitor of AKT. Activated AKT then phosphorylates HEXIM, and LTR induction ensues. Vaccination may activate some T cells, inducing transcription from quiescent HIV LTRs. (b) Clearance. Once viral transcription has been induced, cells containing HIV must be cleared. There are several mechanisms that may contribute to clearance. If HIV transcription is sufficient to produce HIV protein, HIV antigens may be displayed on the cell surface, which may allow the immune system to target and destroy the infected cells. Alternatively, the cell may undergo apoptosis in response to HIV production. Antiretroviral therapy (ART) will be used to prevent de novo infection. 
a

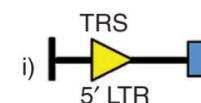
HIV genes (1)

ii)

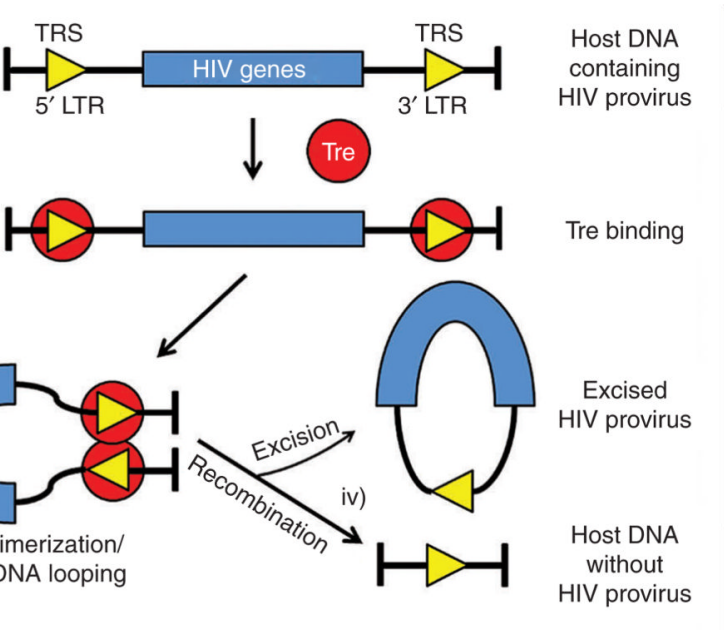

b

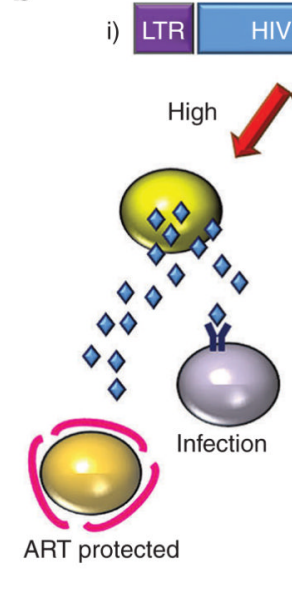

i) LTP ii)

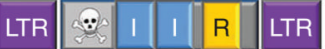

High
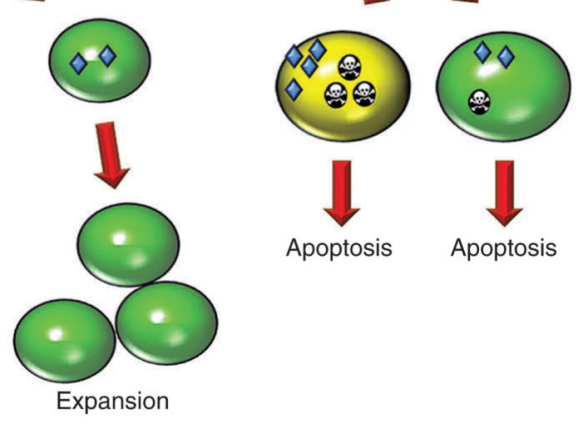

Figure 2.

Gene therapy approaches to eradication: (a) HIV-1-specific recombinase. (i) The HIV provirus contains a sequence within each long terminal repeat (LTR) that is recognized by Tre recombinase. (ii) Tre is introduced into the cell and binds the TRS. (iii) Tre dimerizes and loops out the HIV proviral DNA. (iv) The DNA is recombined, resulting in excision of the intervening sequence containing the provirus and religation of the provirus-free host DNA. (b) Suicide genes. (i) High-level induction of HIV proviral expression can lead to a flood of infectious viral particles. In the presence of antiretroviral therapy (ART), most de novo infections will be prevented, whereas in the absence of sufficient ART, infection can occur. Alternatively, low-level induction may fail to produce sufficient viral proteins for the immune system to be able to identify the infected cell, allowing these cells to persist and possibly expand by homeostatic proliferation. (ii) HIV-dependent suicide gene constructs encoding toxic or proapoptotic genes driven by the HIV LTR and regulated by inhibitory sequences from HIV gag (I) and a Rev response element (R) result in cell death on expression of HIV Tat and Rev, while sparing uninfected cells. TRS, Tre recognition sequence. 


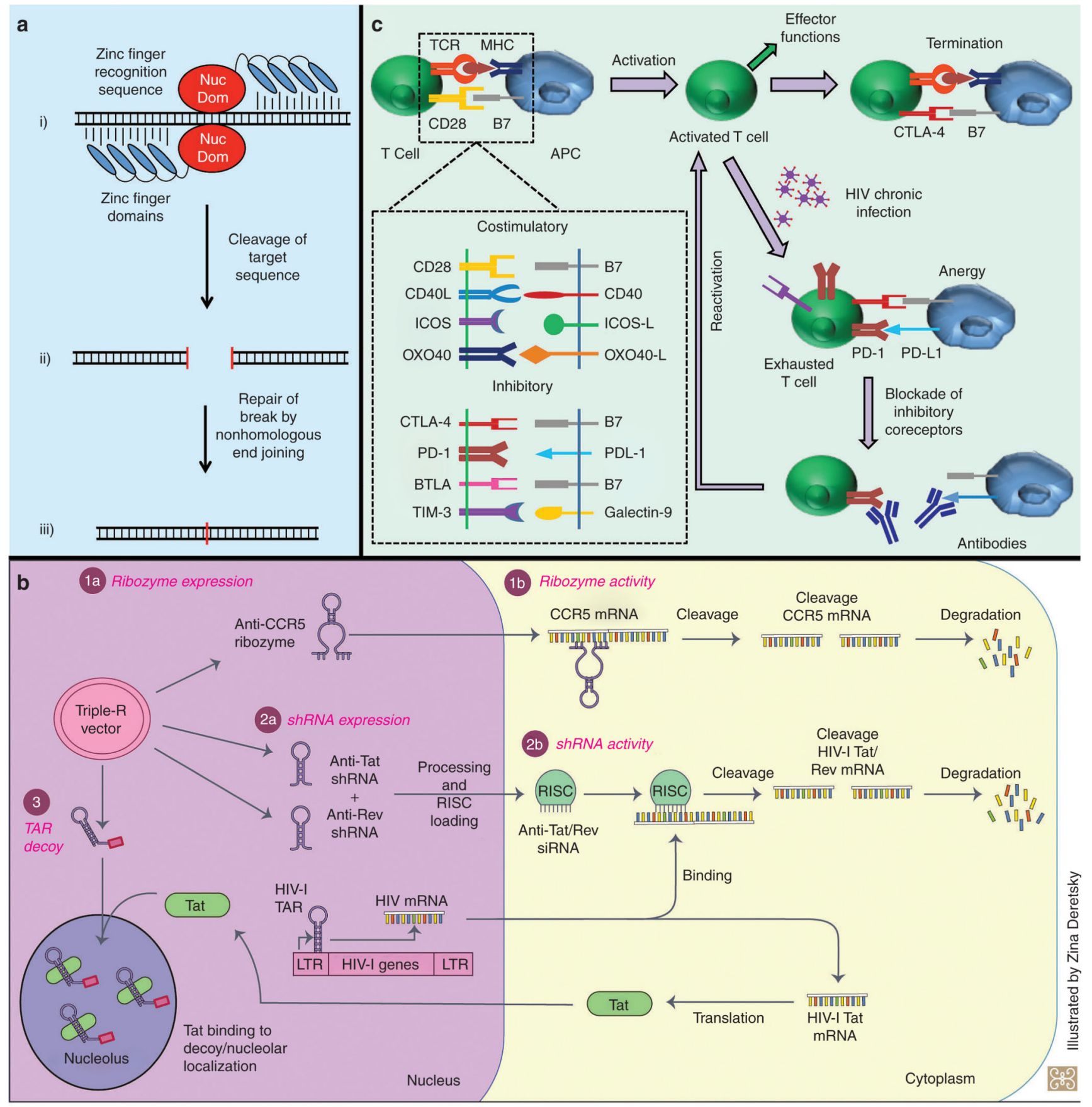

Figure 3.

Strategies to control persistent infection: (a) Zinc-finger nucleases (ZFNs). (i) CCR5specific ZFNs bind sequences within the $C C R 5$ gene, forming a ZFN heterodimer around a central target sequence. (ii) The ZFN nuclease domains (Nuc Dom) cleave the CCR5 gene, creating a DNA double-strand break (DSB). (iii) The DSB can be repaired by homologous recombination (not shown) or nonhomologous end joining, which often leads to deletions at the break site and a reading frame shift in the encoded messenger RNA (mRNA) that gives rise to nonfunctional CCR5 protein. (b) Triple-R expression. The Triple- $R$ vector encodes 1a (an anti-CCR5 ribozyme), 2a (short hairpin RNAs targeting Tat and Rev), and 3 (a 
transactivation response element (TAR) decoy containing a nucleolar-localization tag). $1 \mathrm{~b}$, The ribozyme binds CCR5 mRNA, cleaves it, and then RNA is degraded. $2 b$, The short hairpin RNAs are processed into small interfering RNAs (siRNAs) and the guide strand is loaded into RNA-induced silencing complex (RISC). Upon binding of the siRNA/RISC to Tat or Rev mRNA, the mRNA is cleaved and degraded. 3, Tat protein that re-enters the nucleus is bound by the TAR decoy and sequestered at the nucleolus, thus titrating it away from HIV TAR. (c) Reversal of immune exhaustion using antibodies against inhibitory receptors. Costimulatory and inhibitory molecules are expressed on both T-cell and antigenpresenting-cell surfaces. T cells exert their specific effector functions by engagement of costimulatory molecules, and the immune response is terminated due to expression of inhibitory receptors. During chronic HIV infection, in which the immune system is continuously exposed to the antigen, inhibitory receptors are overexpressed, and the cells become anergic. The use of monoclonal antibodies directed against inhibitory receptors, or their ligands, may restore T cells' capacity to develop their specific immune functions. 


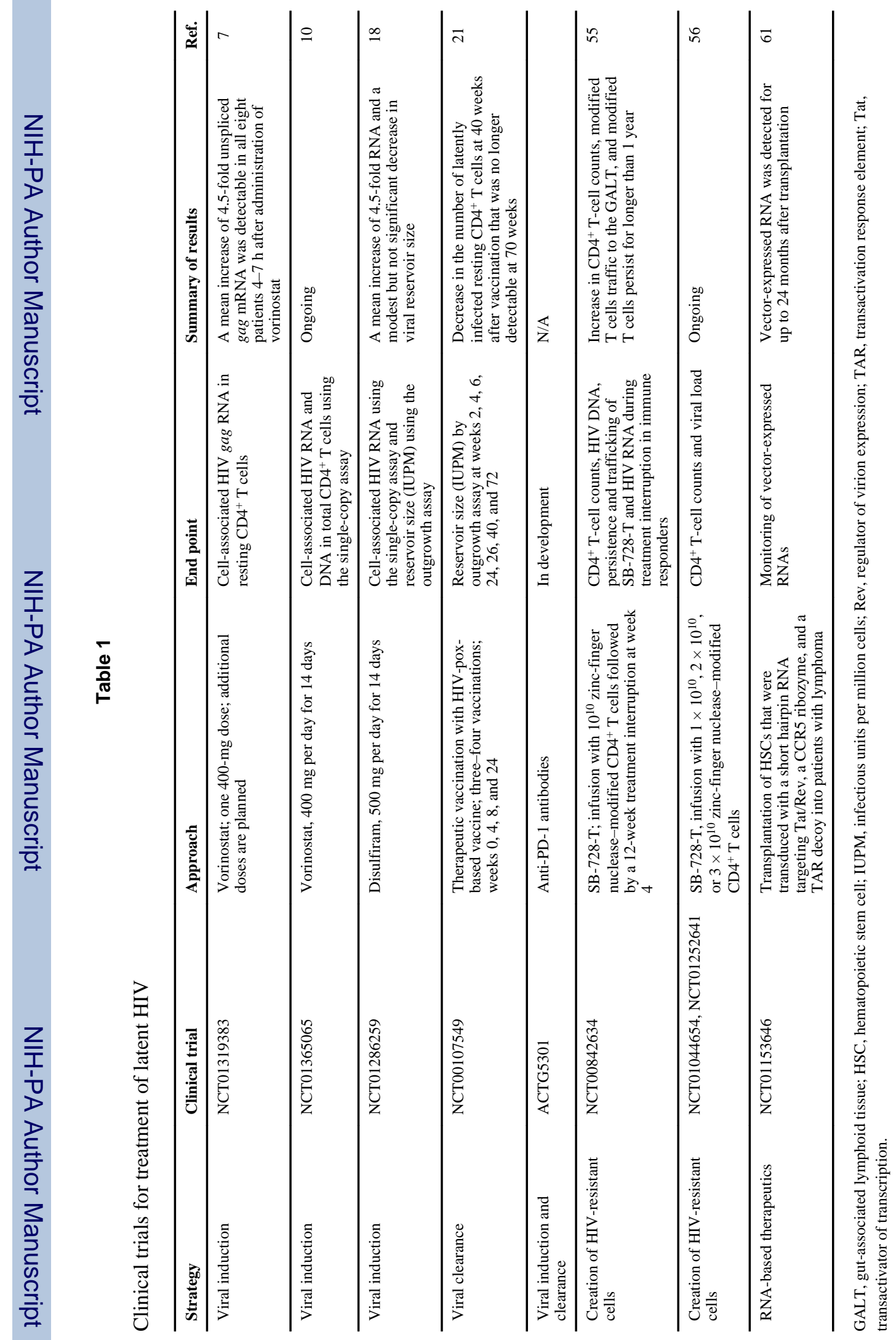

